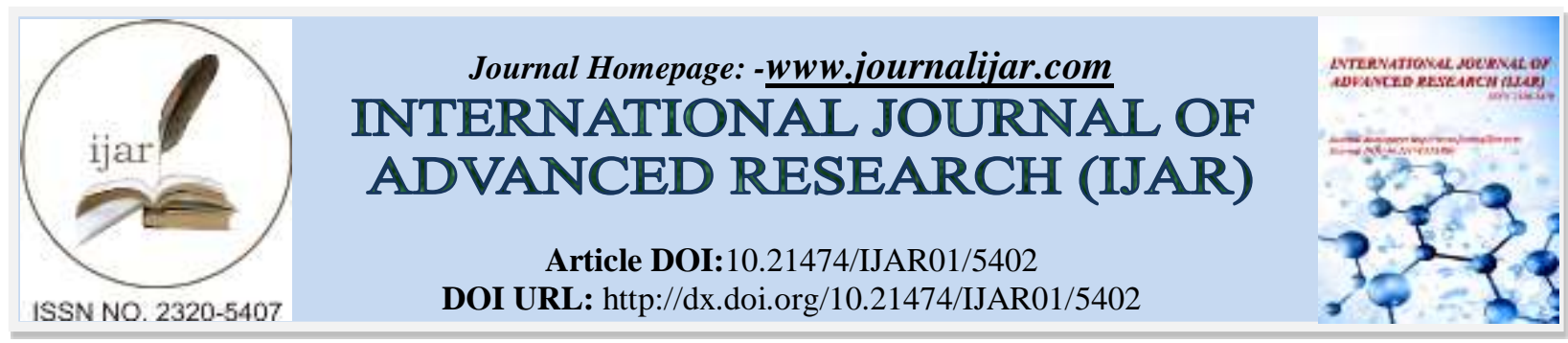

RESEARCH ARTICLE

\title{
ASSOCIATION AND INTERACTION OF POLYMORPHISMS IN ANGIOGENESIS GENES INVOLVED IN ENDOMETRIOSIS.
}

\section{LathaPh.D ${ }^{1,2,5}$, S.Govindan Ph.D ${ }^{1}$,S.N.Ahmad Ph.D ${ }^{1,3}$, S Vaidhya Ph.D ${ }^{1,3}$,C S MovvaPh.D ${ }^{1}$, V.Kutala $\mathbf{P h D}^{2}$, and Q.HasanPh.D ${ }^{1,3,4}$ and V.KodatiPh.D ${ }^{1,2}$.}

1. Dept of Genetics, Vasavi Medical and Research Centre, Khairtabad, Hyderabad- 500 004, Telangana, India.

2. Department of Biochemistry, Nizams Institute of Medical sciences, Hyderabad, Punjagutta, Hyderabad, Telangana, India.

3. Dept of Genetics, University College of Science, Osmania University, Hyderabad-500 007, Telangana, India

4. Dept of Genetics and Molecular Medicine, Kamineni Hospitals, L.B.Nagar, Hyderabad-500 044, Telangana, India.

5. Department of Pathology, JIMS Homeopathic Medical College and Hospital, Sriramnagar, Shamshabad, Telangana, India.

\section{Manuscript Info}

Manuscript History

Received: 11 July 2017

Final Accepted: 13 August 2017

Published: September 2017

Key words:-

Endometriosis, Angiogenesis, Matrix metalloproteinase-2, Matrix metalloproteinase-9, Intercellular Adhesion molecule-1.

\begin{abstract}
Endometriosis is a multifactorial disorder where angiogenesis or neo visualization plays an important role in the etiopathogenesis of the disease, which has a prevalence rate of $10-15 \%$ in women of reproductive age. Polymorphisms in functionally relevant candidate genes involved in angiogenesis may be important for identifying individuals at high risk of developing endometriosis. Hence, three single nucleotide polymorphisms in Matrix metalloprotienase-2 (rs243865), Matrix metalloprotienase-2-9 (rs3918242) and Intra cellular adhesion molecule-1 (rs1799969) genes were evaluated in individuals with endometriosis and compared with sex and age matched controls.. The present study indicates significant difference in allele distributions of the three SNPs between endometriosis and controls. In the case of ICAM-1 (-G241A) a protective association of GA genotype with endometriosis was seen. Clinical features like dysmenorrhea/pain and infertility were associated with MMP2 and MMP9 polymorphism but ICAM-1 genotypes are directly associated with the pain but not with infertility. According to MDR analysis MMP-2, MMP-9 and ICAM-1 genes were individually contributing their role in causing the disease without interacting with each other. Hence these three SNPs can be used for risk assessment of endometriosis in South Indian population.
\end{abstract}

Copy Right, IJAR, 2017,. All rights reserved.

\section{Introduction:-}

Endometriosis is a chronic, progressive disease that frequently presents with multiple sites of extra-uterine tissue in the peritoneal cavity, it affects approximately $10 \%-15 \%$ of women during their reproductive years. The most widely accepted hypothesis of its aetiology is retrograde menstruation followed by angiogenesis for its progression (1). This phenomenon describes that the endometrial cells spill into the peritoneum, which usually get cleared by the immune 
system, while, in some women, the cytokines and growth factors produce local inflammatory reaction which enables the endometrial cells to proliferate and develop angiogenesis so as to promote endometriosis. Hence angiogenic factors are considered to play a significant role in the initiation and progression of the disease which causes debilitating pain, infertility and severely affects quality of life with symptoms mimicking those associated with other chronic pain disorders, such as irritable bowel syndrome, pelvic inflammatory disease etc.

Degradation of basement membrane (BM) is a central phenomenon for angiogenesis, as it allows migration of proliferating endothelial cells (ECs) so as to promote the formation of neo-vessels and plays an important role in the pathophysiology of endometriosis. Accumulating evidence has shown that the peritoneal fluid of endometriotic patients contains more angiogenic factors than that of women without the disease (2). A study has provided evidence that matrix metalloproteinases (MMPs) play a critical role in migration of endothelial cells (3). MMP-2, a member of MMPs, predominantly degrades gelatin and type IV collagen, the major structural component of basement membrane, and thus has been considered as an important factor in invasion and angiogenesis (4). While MMP-9 plays an important 'role in the proteolysis of BM and facilitates the migration of ECs. Other factors including Intercellular Adhesion Molecule-1 (ICAM-1), (5) a macromolecule with immunomodulatory function expressed on a number of cell types including ECs ICAM-1 by virtue of its integrin binding also promotes the activation and release of MMP- 9. $(6,7,8)$.

Polymorphisms in the three candidate genes MMP-9, MMP-2 \& ICAM-1 are considered to be important for the variation seen in polygenic disorders like endometriosis $(9,10)$ where angiogenesis plays an integral part in the growth and establishment of endometriotic lesions. Hence we decided to study the single nucleotide polymorphisms in these three genes which participate in angiogenesis $(11,12,13)$ in MMP-2 (-C1306T), MMP-9 (-C 1562 T) and ICAM-1 (G241A). All these SNPs have been evaluated in different ethinic groups $(14,15,16)$ but have not been assessed in the Indian population.

\section{Methods:-}

Ethical Approval and Consents:-

Approval [VMRC-E-3-2010] for genetic studies in endometriosis was taken from the Ethics Committee of Vasavi Medical and Research Centre. Individual consent was verbal, with the recognition that positive findings would be diagnostically reconfirmed in conjunction with clinical counselling and feedback.

\section{Study participants:-}

In total, 200 pathology-proven endometriosis patients were enrolled into this study during the period 2006-2012. All the patients have accepted to undergo the ultrasonography examination before their biopsies were taken. Disease staging from I to IV was done in accordance with the revised classification criteria set out by American Society for Reproductive Medicine [2000]. Symptoms such as dysmenorrhea, lower abdominal pain, infertility or abnormal menstruation were common to all the patients. Clinical information of patients such as clinical stage, lesion size, location, drug treatment and fertility status was collected from medical records. 200 healthy female controls, without any evidence of endometriosis symptoms were chosen during their attendance for regular gynecological check-ups in the same hospitals. Both patients and controls shared similar age profile.

\section{Sampling and DNA isolation:-}

Approximately $1 \mathrm{ml}$ of venous blood was collected into EDTA containing vacutainer tubes from each subject by venepuncture method. Genomic DNA from blood samples was isolated using conventional salting out procedure published from our lab [27].

\section{Genotyping:-}

Public dbSNP database, NCBI [www.ncbi.nlm.nih.gov/projects/SNP] was used to extract the genotype information for the selected SNPs. PCR for rs243865 (-1306C/T) of MMP2, rs3918242 (-1562 C/T) of MMP9 and of ICAM-1 rs1799969 (G241A) genes was performed using specific oligonucleotide primers synthesized by Bioserve(India) and conditions described in table 1 . Which were slightly modified from the 3 step PCR described earlier by our group (17),193bp PCR amplicon of MMP-2 yields $120 \mathrm{bp}$ and $34 \mathrm{bp}$ bands for "C" allele, while it results a $150 \mathrm{bp}$ band for "A" allele upon the digestion with BfaI enzyme. To identify G241A polymorphism of ICAM-1 gene, allele specific PCR was adopted with a G to T mutated forward primer that introduces a restriction site for BsrGI enzyme, which generates DNA fragments of 90 and 20 base pairs for "A" allele digesting a 160 bp PCR amplicon[Figure 1]. 
For quality control measures, appropriate negative controls were maintained at each step of analysis. Association between individual genotypes and disease manifestations were determined.

\section{Statistical analysis:-}

Hardy-Weinberg equilibrium (HWE) for genotypic distribution was determined with the HWE exact test for each group. $2 \times 2$ contingency tables were plotted based on the presence or absence of the variable in cases and controls. Fisher's exact test was used for the analysis of contingency tables to obtain crude odds ratio (OR) and $95 \%$ confidence interval (CI). Since Endometriosis is perhaps a multifactorial disease, there are several confounding factors that can interfere with statistical analysis. The lower and upper limits for allele frequencies of rs 243865 , rs3918242and rs1799969 polymorphisms were determined using logistic regression analysis, the confounding effects of well established risk factors were controlled and adjusted ORs were obtained, which in turn be useful to predict independent effects. Multifactor Dimensionality Reduction (MDR) analysis was used to study high-order gene-gene and gene-symptom interactions. For this analysis, genotype data computed as 0,1 and 2 based on number of variant alleles. Confounding symptoms were computed as 0 and 1 for the absence or presence of exposure was considered. All the predictor variables were labeled as X1, X2, X3, X4,...... And disease outcome was labeled as Class (MDR version 2.0, beta 6).

\section{In silico analysis (SIFT) and POLYPHEN-2 (Polymorphism phenotyping V2) TOOL:-}

Prediction of tolerant and deleterious mutations /SNPs using SIFT program which can distinguish the functionally neutral and deleterious amino acid changes; hence, it is widely used to detect if an amino acid substitution affects the structure and function of a protein molecule (http://blocks.fhcrc.org/sift/SIFT.html) [Henikoff et al,2001]. Classification of SIFT scores is as follows: damaging $(0.00-0.05)$, potentially damaging $(0.051-0.10)$, borderline (0.101-0.20), or tolerant (0.201-1.00).Simulation for functional change in coding mutations/SNPs by PolyPhen which determines the position and impact of an amino acid substitution on the structure and function of human protein basing on combinational information of phylogenetic, structural, and sequence annotations. It receives amino acid sequence or corresponding ID, position of mutant as input data [Ramensky et al,2002].Classification of PolyPhen score is as follows: probably damaging (>2.00), possibly damaging (1.50-1.99), potentially damaging (1.25-1.49), or benign (0.00-0.99).

\section{Results:-}

The demographic and clinical data of all 409 women was documented. The median age was $33.8 \pm 6.83$ years (range 15 - 54years) in women with endometriosis and 39 5 5.84years (range 20-63years) among controls. The average age at menarche was 14 years (range $11-17$ years) for cases and 12.5 years (range 11-14 years) for controls. Of the cases with endometriosis 7 had menstrual irregularities, 9 were unmarried in the endometriosis group, 6 were post menopausal).

\section{Individual genetic effects:-}

The genotype distribution for all the polymorphisms was in accordance with the Hardy-Weinberg equilibrium in cases and controls. The distribution of variant allele frequencies in cases/controls were as follows: MMP2C1306T (17.5\% vs. $12.3 \%)$, MMP9C1562T (14.2\% vs. $10.1 \%)$, ICAM1 G241A (14.5\% vs. $32 \%)$ Among the three putatively functional polymorphisms tested, the allele frequencies of MMP2C1306T (OR=1.5098, 1.0218-2.2309) and MMP9C1562T $(\mathrm{OR}=1.5979,1.0218-2.2309)$ were independently associated with the endometriosis risk, whereas ICAM1 G241A (OR: $0.3623(0.2562-0.5124)$ conferred protection (Table1, 3).

\section{Gene-gene interactions:-}

The MDR analysis, as well as the logistic regression analysis indicated significant gene-symptom interactions between three allelic variants i.e. MMP2, MMP9, ICAM1 in modulating the endometriosis risk. When the different genotype combinations at MMP2, MMP9 and ICAM1 were examined, adjusting age, infertility and pain/dysmenorrhea for logistic regression, results showed $\mathrm{OR}=2.3112(0.8839-2.0149)$ indicating 2 fold higher risk for endometriosis. But presence of ICAM1 G allele alone seems to be protective in action (Fig 1).

\section{In Silico Analysis:-}

Output reveals of different types of in silico testing of the three identified angiogenic gene variants shows that ICAM-1 with 0.01 SIFT score predicts that the change damages the structure and function and Polyphen score reveals that it is benign. Whereas MMP2 and MMP9 does not show any prediction result. 


\section{Risk predictionmodel:-}

Sensitivity and specificity were calculated using Fisher's exact test. Receivers operating curve (ROC) was plotted using1-specificity on X-axis and sensitivity on Y-axis for all the predictor variables. In subjects without protective alleles, $>4$ cut-off of risk alleles clearly discriminated cases and controls with a sensitivity of 0.76and a specificityof1.00 (Figure-1).In subjects with three protective alleles, none of the risk alleles showed statistically significant association. The ROC curve, $\mathrm{C}$ value being $\mathrm{C}=0.68$ and $\mathrm{C}=0.67$ indicates moderately discriminatory ability in subjects with one Protective allele and $\mathrm{C}=0.57$ indicates mild and poor risk discriminatory ability in subjects towards the endometriosis disease.

Table-1:-Analysis in angiogenic gene polymorphisms among the endometriosis cases and controls.

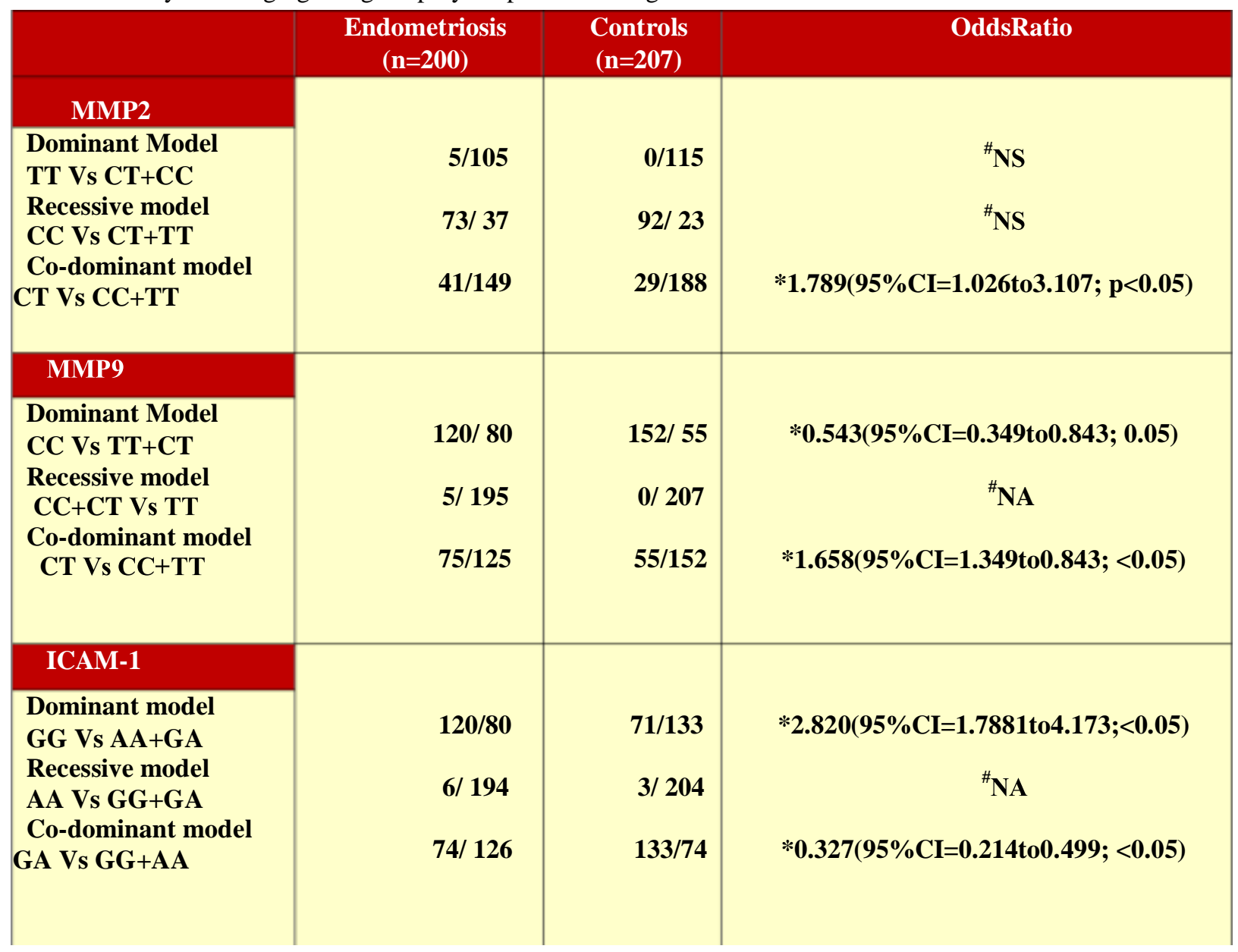

Table-2:-Univariant analysis in angiogenic gene polymorphisms among the endometriosis cases and controls.

\begin{tabular}{|c|l|l|l|l|l|l|}
\hline MMP2C1306T & $141(70.5 \%)$ & $41(20.5 \%)$ & $8(4 \%)$ & 0.17 & $1.5098(1.0218-$ & \\
Cases Controls & $167(80.6 \%)$ & $29(14 \%)$ & $11(5.4 \%)$ & 0.12 & $2.2309)$ & $<0.05^{*}$ \\
\hline MMP9C1562TCases & $123(75.5 \%)$ & $75(20.5 \%)$ & $5(4 \%)$ & 0.14 & $1.707(1.59-2.516)$ & \\
Controls & $152(80.6 \%)$ & $55(0.4 \%)$ & $0(1.93 \%)$ & 0.10 & & $<0.05^{*}$ \\
\hline ICAM-1G241A & $120(60 \%)$ & $74(37 \%)$ & $6(3 \%)$ & 0.14 & $0.3623(0.2562-$ & \\
Cases Controls & $71(34.2 \%)$ & $133(60.9 \%)$ & $3(1.44 \%)$ & 0.31 & $0.5124)$ & $<0.05^{*}$ \\
\hline
\end{tabular}

Table-3:- The distribution of three functionalgenetic polymorphismsamongEndometriosis casesandcontrols.

\begin{tabular}{|l|l|l|}
\hline MMP2 & $0.8786(0.5407-1.4276)$ & NS \\
\hline MMP9 & $1.1238(0.7434-1.6987)$ & NS \\
\hline ICAM-1 & $2.1382(1.2362-3.6982)$ & $0.006^{*}$ \\
\hline
\end{tabular}


AdjustedOR:oddsratioadjustedforage, bodymassindex,ageatmenarche,parity,familyhistoryofendometriosis,CI:confid enceinterval, ${ }^{*}: \mathrm{P}$ value statistically significant $(<0.05)$.

Table4:-Gene-gene interactions between three genes of angiogenic pathway modulating Susceptibility to endometriosis MMP2 and ICAM-1 in combination with MMP9.

\begin{tabular}{|l|l|l|l|}
\hline MMP2 MMP9 ICAM-1 & & Controls & Adjusted OR(95\%CI) \\
\hline W-W-W & 84 & 49 & 1 (REF) \\
\hline M-W-M & 11 & 17 & 0.035(0.151-0.936)* \\
\hline M-M-W & 12 & 8 & NS \\
\hline W-W-M & 25 & 90 & $\mathbf{0 . 1 6 2 ( 0 . 0 0 8 8 - 0 . 2 9 0 ) *}$ \\
\hline W-M-W & 32 & 13 & NS \\
\hline M-W-W & 33 & 12 & NS \\
\hline M-M-M & 3 & 7 & NS \\
\hline M-M-M & 11 & 14 & NS \\
\hline
\end{tabular}

W: Wild genotype, M: heterozygous and homozygous mutant genotypes, adjusted OR: Odds ratio adjusted for age, age of menarche, parity, stage of the disease, pain. CI: Confidence interval, ref: reference genotype combination value statistically significant. Note: The data obtained by MDR analysis was reconfirmed using logistic regression to demonstrate the risk pattern across different genotype combinations.

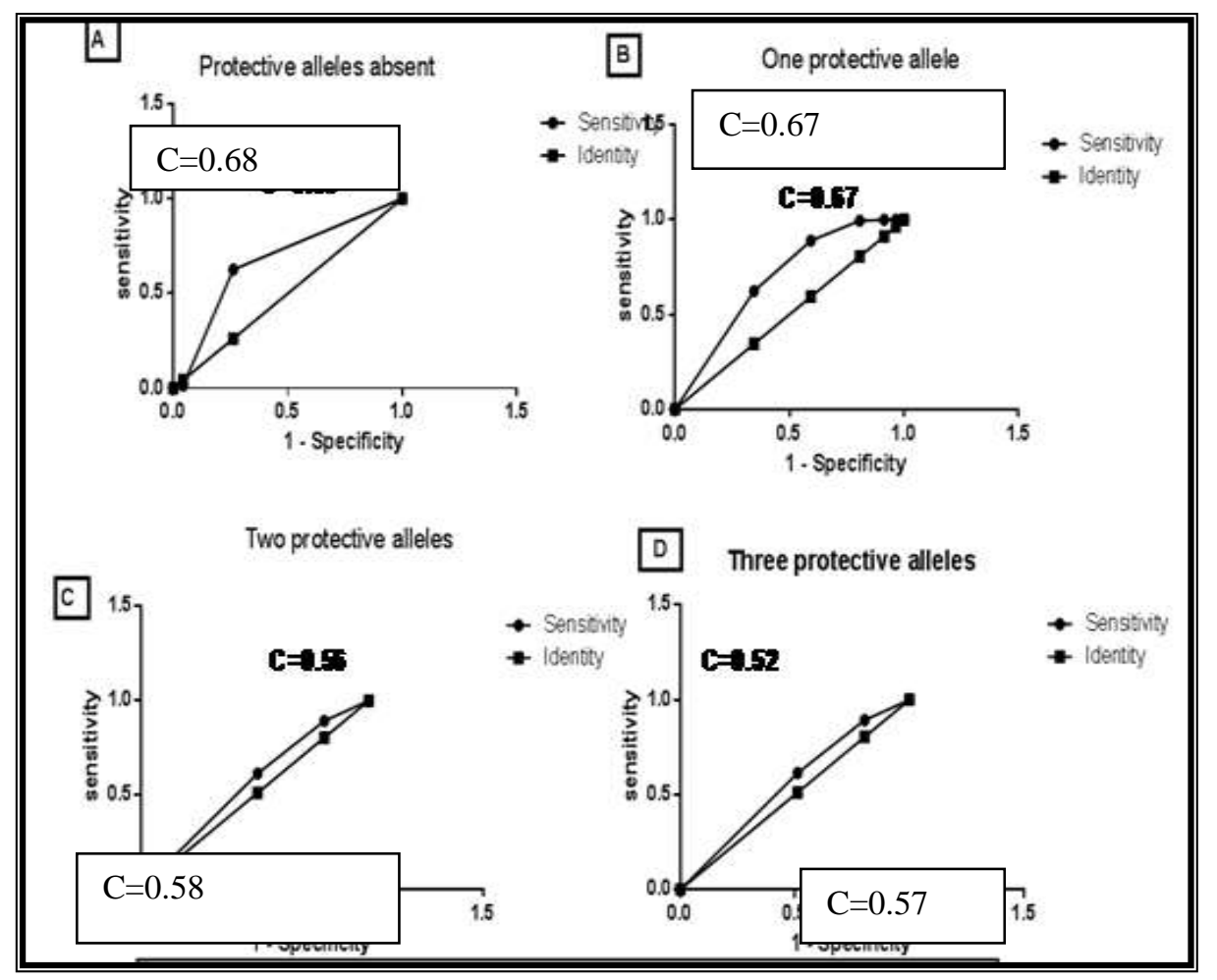

Figure 1:- Risk prediction model of endometriosis by using protective alleles of Angiogenic pathway.

Reciever operating characterstic (ROC) curves for endometriosis prediction model. A,B,C and D represent ROC curves (Sensitivity Vs 1-Specificity) for high risk allele when protective alleles are 0,1,2 and3 (1+2). The area under the curve (C) was used to discriminate the cases and controls. The $\mathrm{C}=0.68,0.67$ for $\mathrm{A}, \mathrm{B}$ curves represents moderate discrimination risk towards the disease wher as $\mathrm{C}$ and $\mathrm{D}$ curve with 0.56 and showed poor risk predictability.

\section{Discussion:-}

There is a growing evidence that the endometrium of women with endometriosis is not nomal and it is suggested that the endometrium has enhanced proliferation and increased ability to implant and survive in ectopic locations. This concept suggests that angiogenesis is essential if endometrium has to survive outside the uterus. Theories 
proposed to account for this susceptatiblity include genetic predisposition(18), a greater amount of retrograde menstruation(19), an altered peritoneal environment (20),immunological susceptibility or an Infectious factor (21 ).

We propose that women develop endometriosis because of abnormalities inherent in their ectopic/intrauterine endometrium. Endometrium is a unique adult tissue which undergoes intense proliferation, secretion, regression and regeneration during each menstrual cycle. It is plausible that subtle alterations in this complex series of events could lead to pathological proliferation of endometrial tissue initiate angiogenesis at ectopic sites. Our hypothesis is that the women with Endometriosis have an altered capacity due to specific gene polymorphisms and therefore implant and which leads to growth in the peritoneal cavity.

Angiogenic factors like interleukins and pro-inflammatory cytokines, cause proliferation and migration of endothelial cells with neovascularisation promotes implantation of the endometrial tissue of ectopic sites. This requires BM degradation by zinc binding proteases called matrix metalloproteinase's (MMPs) especially MMP-2 and MMP-9 which are triggered by molecules like Vascular Endothelial Growth Factor (VEGF) and ICAM-1. ICAM-1 belongs to an immunoglobulin class of adhesion molecules expressed on ECs lining the blood vessels The interaction of ICAM-1 with integrins promotes the activation and release of MMP-9. (9,11). Therefore, both MMP-9 and ICAM-1 genes regulate the downstream pathway of angiogenesis.

Several single nucleotide polymorphisms (SNPs) in the $M M P-2$ promoter region have been identified. Among them, a $\mathrm{C}$ to $\mathrm{T}$ transition located at nucleotide -1306 abolishes a Sp1-binding site and consequently diminishes promoter activity indicating the functional significance of this polymorphism (22). Price et al showed that the $-1306 \mathrm{C} / \mathrm{T}$ cause strikingly low promoter activity, because it disrupts a Sp1-binding site (CCACC box). Because Sp1 is a ubiquitously expressed transcriptional factor that regulates a variety of gene in a constitutive or inducible manner $(13,26-28)$, it is clear that sequence variations may alter the level and specificity of gene transcription Accumulated evidence has shown that the $\mathrm{C} / \mathrm{T}$ transition at this site could lead to lower levels of MMP-2 protein in individuals carrying the $\mathrm{T} / \mathrm{T}$ genotype than in those carry the $\mathrm{C} / \mathrm{C}$ or $\mathrm{C} / \mathrm{T}$ genotype. Our results indicate that the ' $\mathrm{T}$ '- allele is associated (OR is $1.5098(95 \% \mathrm{CI}=1.0218-2.309))$ with women suffering with endometriosis

Previous functional studies using promoter-reporter assays indicated a, 50\% higher promoter activity of ' $\mathrm{T}$ ' allele compared to ' $\mathrm{C}$ ' allele (23). This is because MMP-9 gene harbors a 9bp sequence (GCGCAC/TGCC) at the promoter, which is an important regulatory element for binding of transcription repressor protein, and this region includes the polymorphic site. Results of MMP-9 indicate that 'TT' genotype is present with a higher frequency in women with endometriosis in our population $(\mathrm{OR}=1.5979$ 95\% $\mathrm{CI}=1.0365-2.4633)$. Clinical analysis of endometriosis patients with genotypes of MMP-9 C1562T polymorphism showed that CT heterozygotes and TT homozygotes were significantly higher among cases with dysmennorhea, /pain and infertility. The five cases, which were homozygous for MMP-9 ' $\mathrm{T}$ ' allele, suffered from severe manifestations of endometriosis with chocolate cysts and adhesions. Three of five cases with TT homozygosity also had concurrent fibroids. The results thus indicate that ' $\mathrm{T}$ ' allele (OR-1.5979, CI=1.0365-2.4633) possibly contributes to progression of the disease to severity and also increases the likelihood of other related gynecological pathologies. Therefore, women with $1562 \mathrm{~T}$ allele with a higher activity of MMP-9 may be at greater risk of developing endometriosis. . Presence of ' $\mathrm{T}$ ' nucleotide abolishes the binding site, thereby preventing the repressor protein binding and increasing the transcriptional activity of MMP9. Over expression of this protein subsequently leads to excessive ECM degradation facilitating endothelial cell migration and angiogenesis essential for endometriosis.

To the best of our knowledge there is only a single study published about association of MMP-9 C1562T polymorphism with endometriosis and in this analysis of genotypic distribution with clinical symptoms was not carried out (16). In conclusion, T allele of MMP-9 can be considered as a potential risk marker for endometriosis in Asian Indian Women. Additionally, the presence of $\mathrm{T}$ allele in patients with endometriosis is significantly associated with classical symptoms of endometriosis like dysmenorrhea and infertility.

ICAM-1 G241A polymorphism has been studied in Italian and Japanese women with endometriosis (13,15). The latter showed an association of ' $\mathrm{A}$ ' allele with endometriosis, while no association of this polymorphism was found in Japanese women. Contradictory to this, our results indicate a higher incidence of GG genotype in patients (60\%) than controls $(35.5 \%)$ while GA genotype was higher among controls $(63 \%)$ compared to patients $(37 \%)$. These results suggested that GG genotype is associated with the development of endometriosis, while heterozygous GA possibly confers protection against this pathology. The $\mathrm{G} \rightarrow \mathrm{A}$ change leading to missense codon 241 (position 721 
at exon 4) results in amino acid change from glycine to arginine in the ligand-binding domain III of ICAM-1. From the results, it is hypothesized that presence of glycine probably results in enhanced ligand binding affinity, cell adhesion and co-stimulatory activities of ICAM-1 molecule thereby affecting the key processes contributing to endometriosis. While heterozygous GA (glycine-arginine) alters the ligand binding efficacy thereby affecting the ICAM-1 mediated immunologic and angiogenic function. Results of ICAM-1 indicate a protection of GA genotype with endometriosis $(0.557,95 \% \mathrm{CI}=0.4443-0.6983)$.

Clinical features like age at menarche, diet, age, infertility, and pain were checked but risk of infertility and pain is highly associated with the disease development compare to other clinical features. (Table-1). Earlier studies have not correlated the ICAM-1 genotypes with disease symptoms. However, one study reported the association of ICAM-1 'A' polymorphism with severe Grade IV endometriosis (15).

ICAM1 is found to be one of the key molecules in lymphocyte infiltration into thyroid gland and literature reveals that high concentration of soluble ICAM-1 correlates with the clinical activity and treatment of hyperthyroidism and Graves disease (24). Probably this could be the reason why homozygosity for ' $A$ ' allele was rare among our study subject. Even MDR analysis shows that ICAM1 acts as hypostatically and pain, infertility plays epistatical with risk of endometriosis. The overall results of the present study indicate that ICAM-1 GG genotype is a risk marker for endometriosis while GA confers protection against the disease.

Candidate gene polymorphism studies are helpful in developing disease markers, particularly in the case of polygenic and multifactorial like endometriosis diseases. MMP-2 C1306T, MMP-9 C1562T polymorphism have been studied in patients with endometriosis from China (25). Given that susceptibility genes may have different effects in ethnically distinct populations, and / or varying effects depending on allele frequencies, it is possible that this polymorphism is associated with endometriosis in Indian women.

Statistical analysis of logistic regression with clinical features indicates that women with endometriosis are 3 times more likely to suffer with dysmenorrheal/pain $[\mathrm{OR}=3.0962,95 \% \mathrm{CI}=1.9450-4.9287]$ and 2 times more likely to have infertility chances $[\mathrm{OR}=2.3112(95 \% \mathrm{CI}=1.2212-4.3742)]$. While women with GG genotype are less likely to exhibit infertility indicating that AG, AA genotypes and G allele possibly enhance ICAM1 activity thereby leading to the classical symptoms of endometriosis like dysmenorrhea and infertility $[\mathrm{OR}=0.2841$ (95\% CI=0.18440.4376)] (Table-1).

While $\mathrm{G}$ allele in the exon 4 of ICAM-1 gene can be regarded as risk markers for endometriosis, while GA genotype of ICAM-1 is considered to play a protective role against the disease in our population. Results of Insilico analysis using SIFT and Polyphen represents that the amino acid change in ICAM-1SNP is showing deleterious prediction and the two other genes MMP2, MMP9 SNPs do not predict any result. This indicates the vital role of ICAM-1.This study highlights the importance of communicating the disease risk based on genetic profiles beyond Odds ratios in view of earlier studies that showed strong association for disease, but were not of predictive value(26).

The major challenge remains in translating this laboratory derived observation to a clinicalsettingforthepurposeofeitherusingthisinformationaspredictivetestingorfor individual choice to change one's lifestyle or to undertake a medical intervention to reduce disease risk. Multi-locus investigations have advantage over single-locus investigations where genetic risk factor profile can be usedtodeducethesensitivityvs.1specificity graphs for different combination of alleles so that area under the ROC curve can beusedtovalidatethediscriminatoryabilityofacontinuousvariable. Thesestatisticswereappliedinthisstudybyincorporati nganumberofprotectiveallelesasadditional markers that distinguishes genetic riskfactor profile in endometriosis.

The major hallmark of this study was the application of MDR analysis apart from the conventional statistical tests to explore the best combination of genotypes from a given set of data to facilitate better understanding of complex biological interactions. By using matched case-control-pairs, potential confounders such as age, ethnicity, geographical locationandmenopausestatuswerecontrolledatthetimeofenrollmentofthesubjects. Other confounders such as age, age at menarche, parity, and pain were controlled using the logistic regression models.

\section{Conclusion:-}

The T-allele of MMP9 can be considered potential risk marker for endometriosis in Asian Indian Women. Additionally, the presence of T-allele in patients with endometriosis is significantly associated with classical 
symptoms of endometriosis like dysmenorrhea and in fertility. While $\mathrm{G}$ allele in the exon 4 ofICAM1genecanberegardedasrisk markers for endometriosis, while GA genotype of ICAM-1 is considered to play a protectiveroleagainstthediseaseinourpopulation. Hence, MMP2, MMP9and ICAM-1 gene scan be used for risk assessment of endometriosis in our population.

Acknowledgement: Department of Science and Technology (SR/WOS-A/LS-121/2010), India.

\section{References:-}

1. Sampson JA and Albany NY (1927). Peritoneal endometriosis due to menstrual dissemination of endometrial tissue into the peritoneal cavity. Am J Obstet Gynecol.14: 422-469.

2. Richard O. Burney, M.D., M.Sc. ${ }^{a}$ and Linda C. Giudice, M.D., Ph.D. ${ }^{b}$ Pathogenesis and Pathophysiology of Endometriosis. Fertil Steril. 2012 Sep; 98(3): 10.1016/j.fertnstert.2012.06.029.

3. Frederick Schatz, Ozlem Guzeloglu-Kayisli, Sefa Arlier, Umit A. Kayisli, and Charles J. Lockwood ${ }^{*}$ The role of decidual cells in uterine hemostasis, menstruation, inflammation, adverse pregnancy outcomes and abnormal uterine bleeding.Hum Reprod Update. 2016 Jun; 22(4): 497-515.

4. Arda Kucukguven and Raouf A. KhalilMatrix Metalloproteinases as Potential Targets in the Venous Dilation Associated with Varicose Veins Curr Drug Targets. 2013 Mar 1; 14(3): 287-324.

5. Adoujit F, Edouard FP, Yves SP. Bi-Directional Induction of Matrix Metalloproteinase-9 and Tissue Inhibitor of Matrix Metalloproteinase-1 during T-Lymphoma/Endothelial Cell Contact:Implication of ICAM-1. The J of Immunol 1998: 160:2967-73.

6. Hammadeh ME, Fischer-Hammadeh C, Hoffmeister H, Huebner U, Georg T, Rosenbaum P, et al. Fibroblast growth factor (FGF), intracellular adhesion molecule-1 (sICAM-1) level in serum and follicular fluid of infertile women with polycystic ovarian syndrome, endometriosis. 2003; 50:124-30.

7. Somigliana E, Vigano P, Gaffuri B, Guarneri D, Busacca M, Vignali M. Human Endometrial Stromal Cells as a source of soluble Intercellular adhesion Molecules (ICAM). Mol Hum Reprod 1996; 1190-94.

8. Maeda N, Izumiya C, Oguri H, Kusume T, Yamamoto Y, Fukaya T. Aberrant Expression of Intercellular Adhesion Molecule-1 And Killer Inhibitory Receptors Induces Immune Tolerance in Women with Pelvic Endometriosis. FertilSteril 2002; 77: $4: 679-83$

9. Vigano P, Gaffuri B, Somigliana E, Busacca M, Di Blasio A. M, Vignali M. Expression of Intercellular Adhesion Molecule (ICAM) -1 mRNA Protein is Enhanced in Endometriosis versus Endometrial stromal Cells in Culture. Mol Hum Reprod 1998; 4:12: 1150-56.

10. Collette T, Bellehumeur c, Kats R, Maheux R, Mailloux J, Villeneuve M et al. Evidence for an increased release of proteolytic activity by the eutopic endometrial tissue in women with endometriosis and for involvement of matrix metalloproteinase-9. Hum Reprod 2003; 19:6: 1257-64.

11. Vanderstolpe A and Vandersaag PT. Intercellular Adhesion Molecule-1. J Mol Med 1996; 74: 13-33.

12. Hayflick JS, Kilgannon P and Gallatin WN. Intercellular Adhesion Molecule-1. (ICAM-1) Family of proteins. Immunol Res 1998; 17: 313-27.

13. Vigano P, Gaffuri B, Somigliana E, Busacca M, Di Blasio A. M, Vignali M. Expression of Intercellular Adhesion Molecule (ICAM) -1 mRNA Protein is Enhanced in Endometriosis versus Endometrial stromal Cells in Culture. Mol Hum Reprod 1998; 4:12: 1150-56.

14. Collette T, Bellehumeur c, Kats R, Maheux R, Mailloux J, Villeneuve M et al. Evidence for an increased release of proteolytic activity by the eutopic endometrial tissue in women with endometriosis and for involvement of matrix metalloproteinase-9. Hum Reprod 2003; 19:6: 1257-64.

15. Yamashita M, Yoshida S, Kennedy S, Ohara N, Motoyama S, Maruo T. Association Study of Endometriosis and Intercellular Adhesion Molecule-1 (ICAM- 1) Gene Polymorphisms in a Japanese Population. J SocGynecolInvestig 2005; $12: 4$; 267-70

16. Shan K, Lian-Fu Z, Hui D, Na W, Xia J and Yan L. Polymorphism in the promoter regions of the matrix metalle proteinase-7,-9 and the risk of endometriosis and adenomyosis in China. Mol Hum Reprod 2006: 12(1):35-39.

17. Chava $S^{1}$, Mohan V, Pasupuleti N, Latha MM, Khan IA, Upendram P, Kumar A, Ahuja YR, Hasan Q.Evaluation of Aurora-A gene polymorphism and esophageal cancer risk in a South Indian population. Genet Test Mol Biomarkers. 2011 Mar;15(3):185-9. doi: 10.1089/gtmb.2010.0143. Epub 2011 Jan 3.

18. Herati $\mathrm{AS}^{1}$, Zhelyazkova $\mathrm{BH}^{2}$, Butler $\mathrm{PR}^{1}, \mathrm{Lamb} \mathrm{DJ}^{3}$.Age-related alterations in the genetics and genomics of the male germ line. Fertil Steril. 2017 Feb;107(2):319-323. doi: 10.1016/j.fertnstert.2016.12.021. 
19. Babic $\mathrm{A}^{1}$, Cramer DW, Titus LJ, Tworoger SS, Terry KLMenstrual pain and epithelial ovarian cancer risk. Cancer Causes Control. 2014 Dec; 25(12):1725-31. doi: 10.1007/s10552-014-0463-6. Epub 2014 Sep 5.

20. Young $\mathrm{VJ}^{1}$, Brown $\mathrm{JK}^{1}$, Saunders $\mathrm{PT}^{1}$, Duncan $\mathrm{WC}^{1}$, Horne $\mathrm{AW}^{1}$ The peritoneum is both a source and target of TGF- $\beta$ in women with endometriosis. PLoS One. 2014 Sep 10;9(9):e106773. doi: 10.1371/journal.pone.0106773. Govindan S, Ahmad SN, Vedicherla B, Kodati V, Jahan P, Rao KP et al2007 Association of Progesterone Receptor Gene Polymorphism (PROGINS) with Endometriosis, Uterine Fibroids and Breast CancerCancer Biomarkers 3(2) 73-78.

21. Saikawa $Y^{1}$, Price K, Hance KW, Chen TY, Structural and functional analysis of the human KB cell folate receptor gene P4 promoter: cooperation of three clustered Sp1-binding sites with initiator region for basal promoter activity. Elwood PC1995 Aug 8;34(31):9951-61.

22. Zhang B, Ye S, Hermann SM, Eriksson P, de Maat M, Elvas A, et al. Functional polymorphism in the regulatory region of Gelatinase B, gene in relation to severity of coronary artherosclerosis. Circ 1999; 99:1788-94.

23. Brzozowska $\mathrm{M}^{1}$, Kretowski A. [Current views on the etiopathogenesis of goiter in children]. Endokrynol Diabetol Chor Przemiany Materii Wieku Rozw. 2006;12(1):35-43.

24. Kitajima $\mathrm{M}^{1}$, Khan $\mathrm{KN}^{2}$, Harada $\mathrm{A}^{3}$, Taniguchi $\mathrm{K}^{3}$, Inoue $\mathrm{T}^{3}$, Kaneuchi $\mathrm{M}^{3}$, Miura $\mathrm{K}^{3}$, Masuzaki $\mathrm{H}^{3}$.Association between ovarian endometrioma and ovarian reserve. Front Biosci (Elite Ed). 2018 Jan 1; 10:92-102.

25. $\underline{\text { Kraft } \mathrm{P}^{1}}$, Benz PM$, \underline{\text { Austinat M}}, \underline{\text { Brede ME}}, \underline{\text { Schuh K}}$, Walter U, Stoll G, Kleinschnitz C.Deficiency of vasodilator-stimulated phosphoprotein (VASP) increases blood-brain-barrier damage and edema formation after ischemic stroke in mice. PLoS One. 2010 Dec 3;5(12):e15106. doi: 10.1371/journal.pone.0015106.

26. Latha $\mathrm{M}^{1}$, Vaidya S, Movva S, Chava S, Govindan S, Govatati S, Banoori M, Hasan Q, Kodati VL.Molecular pathogenesis of endometriosis; Toll-like receptor-4 A896G (D299G) polymorphism: a novel explanation. Genet Test Mol Biomarkers. 2011 Mar;15(3):181-4. doi: 10.1089/gtmb.2010.0178. Epub 2011 Jan 8. 